\title{
On Merging Business Process Management and Geographic Information Systems: Modeling and Execution of Ecological Concerns in Processes
}

\author{
Xinwei Zhu ${ }^{1}$, Guobin Zhu ${ }^{1}$, Seppe vanden Broucke ${ }^{2}$, Jan Recker ${ }^{3}$ \\ ${ }^{1}$ International School of Software, Wuhan University \\ Luoyu Road 37, Hongshan, Wuhan, Hubei, China \\ ${ }^{2}$ Department of Decision Sciences and Information Management, KU Leuven \\ Naamsestraat 69, B-3000 Leuven, Belgium \\ ${ }^{3}$ Information Systems School, Queensland University of Technology \\ 2 George St, Brisbane QLD 4000, Australia \\ \{xinwei.zhu,gbzhu\}@whu.edu.cn, seppe.vandenbroucke@kuleuven.be, \\ j.recker@qut.edu.au
}

\begin{abstract}
Business Process Management describes a holistic management approach for the systematic design, modeling, execution, validation, monitoring and improvement of organizational business processes. Traditionally, most attention within this community has been given to control-flow aspects, i.e., the ordering and sequencing of business activities, oftentimes in isolation with regards to the context in which these activities occur. In this paper, we propose an approach that allows executable process models to be integrated with Geographic Information Systems. This approach enables process models to take geospatial and other geographic aspects into account in an explicit manner both during the modeling phase and the execution phase. We contribute a structured modeling methodology, based on the well-known Business Process Model and Notation standard, which is formalized by means of a mapping to executable Colored Petri nets. We illustrate the feasibility of our approach by means of a sustainability-focused case example of a process with important ecological concerns.
\end{abstract}

Keywords: geographic information systems, business process management, business process model and notation, sustainable processes, data-aware processes, process modeling, process execution, coloured petri nets

\section{Introduction}

Business Process Management (BPM) has evolved as a holistic, systematic management practice for managing, documenting, modelling, analyzing, simulating, executing and improving end-to-end business processes [1]. BPM considers the use of information systems as a key driver for successful business processes [2] by allowing the monitoring, validation and execution of entire business processes as workflows. 
Much attention within the BPM community has traditionally been given to studying control-flow aspects of business processes, meaning the sequence and ordering in which activities can be performed. In recent years, however, other aspects, or "contexts" within this view, have received an increasing amount of attention [3]. As such, scholars have shifted towards studying various approaches that integrate additional contexts so that processes can be rapidly modified and adapted to (new) external data-governed inputs, such as social aspects, logistic information, or resource and inventory artifacts [4].

One particular context which has been gaining traction and we wish to emphasize in this work is that of "Green BPM" [5]. In managing operational processes, enterprises have traditionally been geared towards optimizing economic imperatives, such as time, cost, efficiency and quality. Whilst doing so, they have been a major contributor to environmental degradation caused by consumption of earthly resources, $\mathrm{CO} 2$ emissions and waste. Consequently, organizations are increasingly encouraged to improve operations from an ecological perspective, working towards making their processes environmentally sustainable. From a BPM perspective, such ecological concerns are another context element that should be merged with traditional approaches towards the modeling, analysis or execution of business processes.

This paper contributes to the existing body of work by proposing a structured and formalized methodology towards enabling sustainable process management. Whilst a great deal of work has already been put towards investigating how this context can be taken into account during the planning, design and modeling of processes, the execution of business processes against this context has received less attention. Such an approach would help to govern and constrain control-flow and process behavior (making a decision to execute a particular activity, for instance) based on ecological aspects during the actual "running" of the process. Naturally, it self-evidently follows that such an approach cannot be constructed in isolation from other information systems that are highly relevant in this context. One type of information system that we are interested in specifically are Geographic Information Systems (GIS) [6]. This is because these systems provide a means to store, manipulate and manage various types of geographical data, and thus are also able to provide information regarding ecologic and resource-based aspects that could be used to describe the ecologically relevant context of a business process.

We proceed as follows. Section 2 provides an overview of related work and preliminaries. Section 3 introduces our new structured process modeling approach based on the Business Process Model and Notation (BPMN) standard in order to show how non-control-flow information can be modeled in an explicit manner when designing business processes. In Section 4, we discuss how the execution of such processes can be supported by introducing a formalized mapping to executable colored Petri nets. In Section 5, we describe a case example showing the feasibility of our approach in the context of sustainable processes, where the link with GIS also becomes evident. Section 6 concludes the paper. 


\section{Background}

\subsection{Related Work}

We regard the environmental context as one of the key variables in the wider setting of a business process. In the layered process context model proposed by Rosemann et al. [3], this context is explicitly included as a separate layer influencing the business process - including factors stemming from ecological aspects and geospatial artifacts. Considering including this context towards making BPM sustainable, we refer to the seminal work of vom Brocke et al. [5], where the notion of "Green BPM" was put forward and a framework for sustainable information systems was presented. Other approaches on the topic of Green BPM exist, but it should be noted that the discussions on Green BPM methods are still in the early stages and so far only a few approaches exist [5, 7-10]. In particular, although works have been presented in order to annotate process models with relevant sustainability information or propose modelling extensions to explicitly include ecological information such as carbon footprint effects [11], we wish to take this a step further by also including such aspects during the execution of process models, as to constrain and govern the control-flow of processes. Houy et al. [8, 9] proposed a semi-automated process improvement approach based on environmental information, by constructing a library of process fragments based on which an optimal process model can be constructed. A downside of this approach is that modelers first need to construct such a collection of feasible process fragments and that the optimization is not performed during execution but rather as a post-hoc analysis. Note that we do not only consider this to be a relevant aspect for organizations aiming to optimize their processes in terms of minimalizing the environmental impact of their activities, but also to optimize their efficiency by taking such environmental information into account in the first place. Consider for example a farming process where the decisions regarding soil preparation, crop selecting, seeding/planting, pest prevention, and harvesting can be based on weather information or ground health indicators about the location of the farming processes.

\subsection{Preliminaries}

\section{Business Process Model and Notation}

A general introduction to BPM is available elsewhere [12]. We focus on the industry standard BPMN [13] to present a structured modeling approach to combine process control-flow aspects (which are supported by standard BPMN) with other contextual information such as geospatial or environmental concerns.

BPMN is a standard for business process modeling that provides a graphical notation for designing business processes, similar to a flowchart. Fig. 1 provides an overview of the BPMN graphical elements that will be used throughout the paper. The graphical constructs are categorized in four categories, these being: 
- Flow objects: events, activities and gateways;

- Connecting objects: sequence flow, message flow and association;

- Swim lanes: pools and lanes (grouping other elements);

- Artifacts: data objects, groups, and textual annotations.

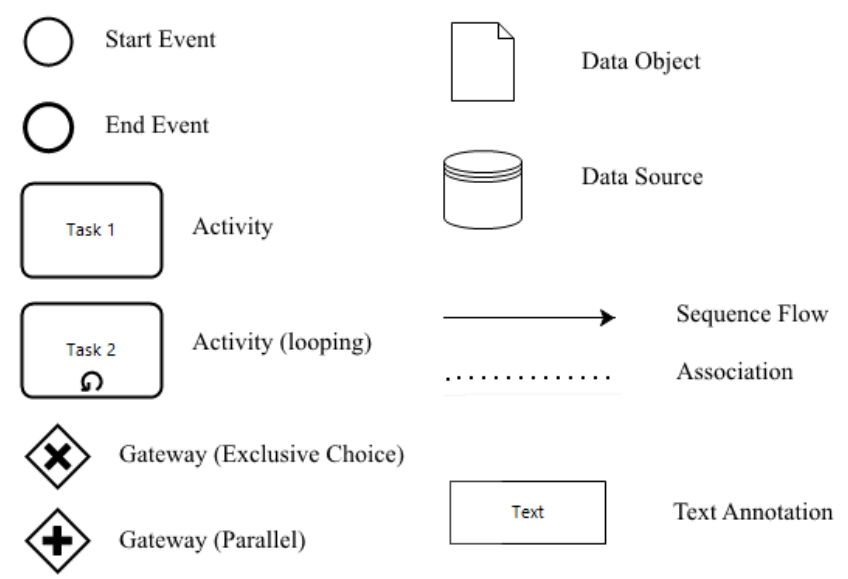

Fig. 1. Overview of common BPMN constructs used throughout the paper.

Every BPMN model starts and ends with a Start Event and End Event element respectively. Sequence Flow arrows connect these elements with a series of Activities. Branching and merging points in the process are represented with Gateway elements, either as an exclusive choice or a parallel branch. Data Objects and Data Sources represent external data which can be associated to sequence elements by means of an Association arc.

In order to examine how structured BPMN can be extended to support geospatial and/or ecological variables, in the following, we base ourselves on earlier published work [14], in which we extended BPMN to support location-dependent patterns. We generalize this here to enable the inclusion of any contextual information using existing BPMN constructs. We discuss this approach in Section 3.

\section{Coloured Petri Nets}

To support the execution of our structured, ecological-aware process models and to support integration with GIS systems, we will provide a formalized mapping from our structured BPMN methodology to coloured Petri nets (CPN) $[15,16]$. CPNs are an extension of Petri nets [17], a mathematical modeling language for the description of distributed systems CPN are the standard formalism to define executable process models and allow for their execution in typical workflow engines.

In the following we briefly review the most important CPN formalisms of relevance to this paper. The interested reader is referred to Jensen et al. [16] for more detailed background information.

Definition 1. A CPN is a tuple (P, T, A, $\Sigma, C, V, N, G, E, M, I)$ with:

- $\quad \mathrm{P}$ the set of places, $\mathrm{P}=\left\{\mathrm{p}_{1}, \mathrm{p}_{2}, \ldots, \mathrm{p}_{|\mathrm{P}|}\right\}$ 
- $\quad \mathrm{T}$ the set of transitions, $\mathrm{T}=\left\{\mathrm{t}_{1}, \mathrm{t}_{2}, \ldots, \mathrm{t}_{\mathrm{T}}\right\}$ with $\mathrm{P} \cap \mathrm{T}=\varnothing$

- A the set of arcs, $A=\left\{a_{1}, a_{2}, \ldots, a_{\mid A}\right\}$

- $\Sigma$ the set of color sets defined within the model A color set is a grouping of colors. A color is an attached value to a token

- $\mathrm{V}$ the set of variables used in the model, $\mathrm{V}=\left\{\mathrm{v}_{1}, \mathrm{v}_{2}, \ldots, \mathrm{v}_{\mathrm{V} V}\right\}$ Note that we indicate the actual value (i.e., color) of a variable $\mathrm{v} \in \mathrm{V}$ as $\mathrm{v}^{*}$

- $\mathrm{C}: \mathrm{P} \cup \mathrm{V} \rightarrow \Sigma$ the function returning the color set associated to a place or a variable in the $\mathrm{CPN}$ model

- $\mathrm{N}: \mathrm{A} \rightarrow \mathrm{P} \times \mathrm{T} \cup T \times \mathrm{P}$ the node function mapping arcs to a place-transition or transition-place flow expression. This function allows for the definition of multiple arcs between the same place-transition or transition-place pair

- $\mathrm{G}: \mathrm{t} \in \mathrm{T} \rightarrow \mathrm{GExpr}$ the guard expression function mapping a transition $\mathrm{t} \in \mathrm{T}$ to a boolean expression (true or false) GExpr denoting whether the transitions is permitted to fire. Evaluating this expression yields a boolean result value, indicated as GExpr* $\in\{$ true,false

- $E: a \in A \rightarrow A E x p r$ the arc expression function mapping an arc $a \in A$ to an expression AExpr. Evaluating an arc expression yields a multiset of tokens, indicated as AExpr $\mathrm{MS}^{*}$ which is to be produced (for transition to place arcs) or consumed (for place to transition arcs). The expression itself can use one or multiple variables in $\mathrm{V}$

The color sets of the input and outputs of the arc expression must correspond to the color sets of the places the arcs connects to: $\mathrm{a} \in \mathrm{A}:\left[\exists \sigma \in \Sigma:\left[\forall \tau \in \mathrm{E}(\mathrm{A})_{\mathrm{MS}}{ }^{*}:[\tau \in \sigma] \wedge \mathrm{C}(\mathrm{P} \cap \mathrm{N}(\mathrm{a}))=\sigma\right]\right]$

- $\mathrm{M}: \mathrm{p} \in \mathrm{P} \rightarrow \mathrm{C}(\mathrm{p})_{\mathrm{MS}}$ the marking function, returning the multiset of tokens contained in a place with $\forall p \in P:[\forall \tau \in M(p):[\tau \in C(p)]]$

- I:p $\in \mathrm{P} \rightarrow \mathrm{IExpr}$ the initialization function, this function initializes places in the model with a state, expressed as colored tokens. The evaluation of an IExpr yields a token multiset, indicated as IExprMs* with $\forall \mathrm{p} \in \mathrm{P}:\left[\forall \tau \in \mathrm{IExpr}_{\mathrm{MS}} *:[\tau \in \mathrm{C}(\mathrm{p})]\right]$

We also define the following functions:

- Let $\mathrm{p}: \mathrm{A} \rightarrow \mathrm{P}$ be a function returning the place attached to an arc, i.e. $\mathrm{p}: \mathrm{a} \in \mathrm{A} \rightarrow \mathrm{P} \cap \mathrm{N}(\mathrm{a})$

- Let $t: A \rightarrow T$ be a function returning the transition attached to an arc, i.e. $\mathrm{t}: \mathrm{a} \in \mathrm{A} \rightarrow \mathrm{T} \cap \mathrm{N}(\mathrm{a})$

- Let Type:A $\rightarrow \Sigma$ be a function returning the type (color set) of the associated place to an arc, Type: $\mathrm{a} \in \mathrm{A} \rightarrow \mathrm{C}(\mathrm{p}(\mathrm{a}))$

The actual execution of CPN models involves "firing" enabled transitions, which move tokens from their input to output places according to the expression on the arcs connecting them.

Definition 2. For a transition $t \in T$ to be enabled, the following criteria need to hold:

- All expressions of the incoming arcs should be satisfied: $\forall \mathrm{a} \in \mathrm{A}, \mathrm{t}(\mathrm{a})=\mathrm{t}:\left[\mathrm{E}(\mathrm{a})_{M S} * \neq \varnothing\right]$

- The guard condition of the transition must evaluate to true, $\mathrm{G}(\mathrm{t})^{*}=$ true 
Enabled transitions can be fired. Output and input places are updated accordingly given the input and output arc expressions. Firing an enabled transition brings a marking M1 $\rightarrow_{t} \mathrm{M}_{2}$ as follows by updating the marking for each connected input and output place. Tokens are removed from input places according to the arc expressions on the arcs connecting those places to the transition which is fired, whereas output places receive tokens according to the arc expressions on the arcs connecting those places to the transition which is fired.

\section{Modeling Contextual Processes}

This section describes a structured BPMN modeling approach in order to define process models with contextual artifacts. These contextual artifacts are in the following represented as instances of data artifacts, whereby the type of data can be defined as any contextual information of relevance, including geospatial or ecological information.

Our approach is structured since we extend BPMN by means of making the approach stricter, following a clear set of guidelines. The following definition provides an overview of these guidelines.

Definition 3. A structured contextual BPMN model is defined as a PBMN model so that:

- Control flow is represented using Start Event, End Event, Activity, Exclusive Choice Gateway, Parallel Gateway and Sequence Flow constructs only (see Fig. 1).

- The Data Source element represents a collection of data elements belonging to the same type. For instance, a Data Source can be defined to represent resources, people, countries, and so on.

- The Data Object element represents a particular data element belonging to a Data Source. This implies that each Data Object has one type.

- Activity elements can involve a number of Data Objects which will be bound to that activity at the time of execution. These Data Objects are connected to the activity by means of an Association arc (from the Activity element to the Data Object element). All Data Source elements are also connected to the Activity Element to indicate the Activity is taking data from the types they represent.

- The execution of Activity elements can be constrained by free-form constraints which are annotated to the Activity element: a Text Annotation connected to the Activity with an Association arc. This allows to govern control-flow at execution time based on external data. Constraints may-but do not have to-involve other Data Objects bound to previous Activity elements, in which case an Association arc is added from the input Data Object to the constrained Activity element. 
Note that the strictness of our approach is not limiting by any means, as any BPMN model can be converted to its structured counterpart (e.g., by merging lanes and pools). In our case example in Section 5, Fig. 2 provides an example of the structured modeling approach, in the form of a build-to-order process.

\section{Executing Contextual Processes}

To support the execution of our structured BPMN process models and to support integration with GIS systems, we now provide a formalized mapping from our structured BPMN methodology to $\mathrm{CPN}$. To be more precise, the conversion from a BPMN model to an executable CPN model is performed as follows.

Definition 4. A structured BPMN model is converted to a CPN as follows:

- $\Sigma=\left\{\mathrm{U}, \mathrm{D}_{1}, \ldots, \mathrm{D}_{\mathrm{n}}\right\}$ with $\mathrm{U}=\{$ unit $\}$ the color set containing on control-flow oriented color and $\mathrm{D}_{\mathrm{i}}=\left\{\mathrm{d}_{1}, \ldots, \mathrm{d}_{|\mathrm{Di}|}\right\}$ a color set for each Data Source contained in the BPMN model with $\mathrm{d}_{1}, \ldots, \mathrm{d}_{|\mathrm{Di}|}$ the Data Elements contained in this Data Source. I.e. Customer $=\{$ customer 1 , customer $2, \ldots\}$

- Control flow elements from the BPMN model (Activities, Sequence Flow and Gateways) can be immediately mapped to CPN models as the semantics of the latter allow all control-flow constructs of the former (i.e. sequence, loop, exclusive choice and parallel split)

- For each Data Source $D_{i}$, an input place $p^{i} D_{i} \in P$ is added to the CPN model with $\mathrm{C}\left(\mathrm{p}^{\mathrm{i}} \mathrm{D}_{\mathrm{i}}\right)=\mathrm{D}_{\mathrm{i}}$ and $\mathrm{I}\left(\mathrm{p}^{\mathrm{i}} \mathrm{D}_{\mathrm{i}}\right)=\left\{\mathrm{d}_{1}, \ldots, \mathrm{d}_{|\mathrm{Di}|}\right\}$

- For each Data Element de $\mathrm{e}_{\mathrm{i}}$ acting as an output for an Activity, an output place $\mathrm{p}^{\circ} \mathrm{de}_{\mathrm{i}} \in \mathrm{P}$ is added to the CPN model with $\mathrm{C}\left(\mathrm{p}^{\circ} \mathrm{de}_{\mathrm{i}}\right)$ equal to the data type corresponding with the Data Element (i.e. the Data Source the Data Element is contained in)

- Constraints in the BPMN model are added as guards to the transitions in the CPN model

- For each Data Element de $\mathrm{i}_{\mathrm{i}}$ acting as an output for an Activity, we add four arcs to the CPN model: two arcs to move a token from an input place $\mathrm{p}^{\mathrm{i}} \mathrm{D}_{\mathrm{i}}$ to the transition and move it back to the input places, an arc to remove a currently bound Data Element from the output place $\mathrm{p}^{\circ} \mathrm{de}_{\mathrm{i}}$ and an arc to assign the chosen Data Element to the output place.

- Whenever a Data Element acts as an input in a constraint of another Activity, we add two arcs from and to the output place containing this Data Element to the transition.

The following section describes a case study which applies our approach in a practical, sustainability-driven context. 


\section{Case Study}

We illustrate the feasibility of our approach against the backdrop of sustainable process modeling by means of a case example. Fig. 2 depicts a structured BPMN model that represents a build-to-order process. The example process model follows a fairly standard and simple sequence of activities in terms of control-flow: whenever an order comes in, the availability for the ordered product is checked. When the product is not available, a job is queued in a manufacturing plant to start production. Once the product is finished (or available), it can be collected and prepared for packaging. Next, a notice is sent to the customer while the product is on the way to be delivered. Once these steps have been taken, the case can be closed.

In addition to simple control flow concerns, the example process model also shows a number of data artifacts which have been taken into account during the modeling of this process, as well as a number of ecological constraints which govern the execution of activities. These are:

(i) an optimal selection plant should be selected to produce a certain product (we leave the definition of "optimal" to the interpretation of the reader, but this can be based on a cost-efficiency-environmental cost tradeoff),

(ii) manufacturing at a plant should start only when a batch is ready to be produced, i.e. when enough products are queued. This is to avoid wasteful startup and overhead costs.

(iii) products should be packaged in an optimal packaging, and finally,

(iv) the delivery to the customer should follow an optimal route, saving fuel costs and consumption. 


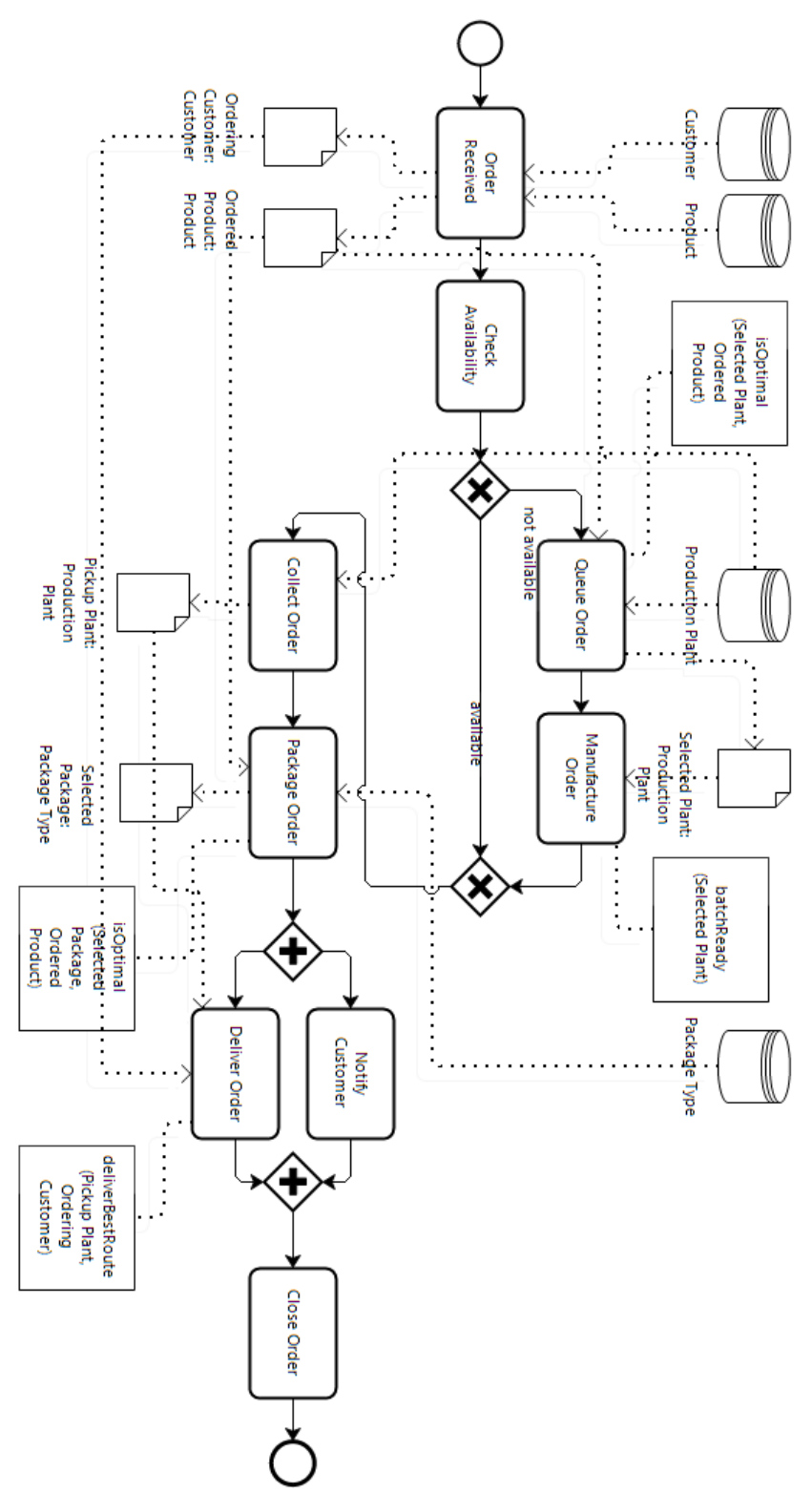

Fig. 2. Example build-to-order process following our structured BPMN approach. 
Fig. 3 shows the result after converting the example BPMN model to an executable CPN model. We have implemented this model using the CPN Tools modeling toolkit [16], and have coupled this with an existing GIS system (based on GeoTools [18]) in order to visualize process-related aspects such as customer locations, delivery teams, production plant status and other geographical aspects. Note that this integration also allows to call the GIS system to calculate the most optimal route for product delivery, taking into account weather and traffic data.

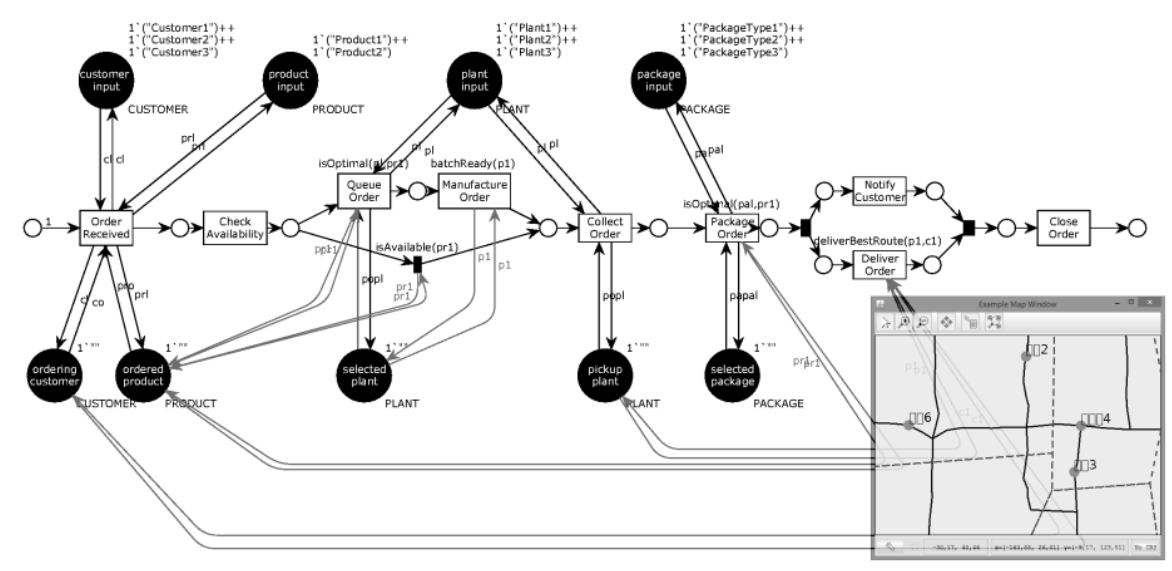

Fig. 3. The example process converted to a running CPN model. The checking of the constraints can be easily coupled with existing GIS systems. In this figure, the GIS system provides a live map (lower right) of deliveries, customers and production plants for running orders. The GIS system can also be called to select the optimal route for delivery, taking into account weather and traffic data.

During execution, the CPN model allows to track process instances as they are running, governed by the ecologic constraints. The system checks these constraints at execution-time and determines which transitions can be fired. In addition, the model approach allows for integration with existing GIS systems, for instance to enable detailed geographical monitoring functionality. This model thus allows analysis of ecological-related concerns and to derive possibilities for improvement.

\section{Conclusions}

We have presented a first approach towards enabling the modeling and execution of processes that can incorporate context information, in our case ecological and geographical concerns. We do so by first proposing a general approach towards constructing process models with any type of contextual data, and then show how to convert this model to a CPN model using a formalized mapping which allows the model to be executable, and to be integrated with GIS systems, for example. This integration with GIS can then perform supporting tasks to "feed" and constrain the 
execution of activities based on geographic data, or the running process can update the GIS system as activities are executed.

In future work, we plan to expand on this initial work by (i) providing mathematical proofs for our formalizations, (ii) designing and executing empirical evaluations using real-life cases, and (iii) exploring the various integration possibilities between GIS and BPM systems in the explicit context of sustainability concerns related to business processes.

Acknowledgments. This work is supported by the National Key Technology R\&D Program, China (grant 2012BAH01F02) and by the KU Leuven research council (grant OT/10/010).

\section{References}

1. Vom Brocke, J. and M. Rosemann, Handbook on Business Process Management: Strategic Alignment, Governance, People and Culture. 2010: Springer.

2. van der Aalst, W.M.P., A.H.M.t. Hofstede, and M. Weske. Business Process Management: A Survey. in Proceedings of the 2003 International Conference on Business Process Management. 2003. Berlin, Heidelberg: Springer-Verlag.

3. Rosemann, M., J.C. Recker, and C. Flender, Contextualisation of business processes. International Journal of Business Process Integration and Management, 2008. 3(1): p. 47-60.

4. Rosemann, M. and J.C. Recker. Context-aware process design: Exploring the extrinsic drivers for process flexibility. in The 18th International Conference on Advanced Information Systems Engineering. Proceedings of Workshops and Doctoral Consortium. 2006. Namur University Press.

5. Vom Brocke, J., S. Seidel, and J. Recker, Green business process management: towards the sustainable enterprise. 2012: Springer.

6. Chang, K.-t., Introduction to geographic information systems. 2010: McGraw-Hill New York.

7. Ghose, A., et al., Green business process management: A research agenda. Australasian Journal of Information Systems, 2010. 16(2).

8. Houy, C., et al. Towards Green BPM-Sustainability and resource efficiency through business process management. in business process management workshops. 2011. Springer.

9. Houy, C., et al., Advancing business process technology for humanity: Opportunities and challenges of green BPM for sustainable business activities, in Green Business Process Management. 2012, Springer. p. 75-92.

10. Recker, J.C., et al., Business process modeling : a comparative analysis. Journal of the Association for Information Systems, 2009. 10(4): p. 333-363. 
11. Recker, J., et al., Modeling and Analyzing the Carbon Footprint of Business Processes, in Green Business Process Management. 2012, Springer. p. 93109.

12. Dumas, M., et al., Fundamentals of Business Process Management. 2013, Berlin, Germany: Springer.

13. Mendling, J. and M. Weidlich, Business Process Model and Notation. 2012.

14. Zhu, X., et al., Exploring Location-Dependency in Process Modeling. Business Process Management Journal, 2014. 20(6).

15. Jensen, K., Coloured petri nets. 1987: Springer.

16. Jensen, K., L.M. Kristensen, and L. Wells, Coloured Petri Nets and CPN Tools for modelling and validation of concurrent systems. International Journal on Software Tools for Technology Transfer, 2007. 9(3-4): p. 213254.

17. Murata, T. Petri nets: Properties, analysis and applications. in Proceedings of the IEEE. 1989.

18. Turton, I., Geo tools, in Open source approaches in spatial data handling. 2008, Springer. p. 153-169. 